\title{
¿DE QUÉ TRATA LA ONTOLOGÍA? UN EXAMEN DE REINHARDT GROSSMANN
}

\section{WHAT IS ONTOLOGY ABOUT? AN EXAMINATION OF REIN- HARDT GROSSMANN}

\author{
Javier Cumpa ${ }^{1}$ \\ University of Miami
}

Recibido: $13 / 10 / 16$

Aceptado: 12/12/16

Resumen: Mi propósito en este escrito es examinar críticamente la ontología de Reinhardt Grossmann. Para ello, investigo en primer lugar su teoría factulista de las categorías. En segundo lugar, discuto su punto de vista fenomenológico sobre el problema de la reducción ontológica de las propiedades, así como también su planteamiento atomista lógico sobre el análisis ontológico de los particulares, las propiedades, el nexo de ejemplificación y los hechos. Por último, exploro su contribución realista al problema de los universales.

Palabras clave: Categorías, análisis ontológico, intencionalidad, universales, hechos.

\begin{abstract}
My purpose in this paper is to critically examine Reinhardt Grossmann's ontology. To do so, I begin by presenting his factualist theory of categories. In the second place, I discuss his phenomenological viewpoint on the problem of the ontological reduction of properties, as well as his logical atomist approach to the ontological analysis of particulars, properties, the exemplification nexus, and facts. Lastly, I explore his realist contribution to the problem of universals.
\end{abstract}

Keywords: Categories, ontological analysis, intentionality, universals, facts.

1. (javiercumpa@me.com) Investigador en la Universidad Complutense de Madrid. Trabaja, sobre todo, en metafísica. También tiene intereseses de investigación en filosofía de la mente y filosofía de la ciencia. Su investigación actual se centra en la relación entre la metafísica y la imagen científica. 


\section{Categorías y propiedades}

Por "teoría de propiedades", Grossmann entiende una teoría que trata de propiedades de individuos. ${ }^{2}$ Según él, hay también una teoría de las categorías, que trata de propiedades de no-individuos. El objeto de la teoría de las propiedades es la categoría de propiedad. Una categoría central adicional en este contexto es la de individuo. Con ella constituye la distinción ontológica fundamental de la teoría por estar presupuesta en todas las clasificaciones ordinarias y científicas. Pero al descubrirse, se descubre también una ley entre diferentes categorías, que llama "nexo de ejemplificación", por la cual no hay individuos sin propiedades. ${ }^{3}$ En la teoría de las categorías de Grossmann hay tres tipos de propiedades: atributos ordinarios, propiedades de propiedades y categorías o propiedades categoriales. Los atributos ordinarios son propiedades y relaciones de individuos. Las propiedades son las cualidades que constituyen las dimensiones de nuestros cinco sentidos. Las relaciones son las que constituyen las localizaciones espacio-temporales de los individuos, tales como 'estar a la izquierda de,' 'estar entre,' 'ser antes de, 'simultáneamente' o 'después de.' Las propiedades de propiedades son atributos de los atributos ordinarios de los individuos. Un ejemplo de propiedad de propiedades es, por ejemplo, 'ser un color.' Un ejemplo de atributo de relaciones es, por ejemplo, 'transitividad.' Aunque estas relaciones son atributos ordinarios de individuos, no pertenecen a la categoría de propiedad, sino a la categoría de relación, porque, a diferencia de las propiedades, los individuos no las tienen a través del nexo de ejemplificación. ${ }^{4}$ Las categorías son siete: particulares, estructuras, universales, hechos, relaciones, clases y número. Ahora bien, a Grossmann le parecen insatisfactorios dos aspectos de esta tipología de las propiedades basada en la división de tipos u órdenes que procede de la teoría de tipos de Bertrand Russell. El primero es que deja fuera categorías o, también, que es incompleta. Hay entidades que no son ni individuos ni propiedades de individuos, tales como conjuntos, hechos o números. ${ }^{5} \mathrm{El}$ segundo es que las relaciones entre las categorías no parecen estar estratificadas como en la de la división de los tipos u órdenes. Para Grossmann, puesto que la percepción sensible nos da a conocer todo tipo de entidad al

2. R. Grossmann, Ontological Reduction, Bloomington, Indiana University Press, 1973, p. 46 y 181 . De ahora en adelante, $O R$.

3. R. Grossmann, The Categorial Structure of the World, Bloomington, Indiana University Press, 1983, p. 5. De ahora en adelante, CSW.

4. Para Grossmann todas las relaciones son enlaces (ties), es decir, relaciones que no necesitan una relación para relacionarse con lo que relacionan o, también, que relacionan directamente.

5. CSW: 224.

Thémata. Revista de Filosofía $\mathrm{N}^{\circ} 56$ (2017) pp.: 213-234. 
conocer los estados de cosas (o hechos) ${ }^{6}$ de nuestra experiencia cotidiana, parecería haber un descenso de todas las entidades de la jerarquía de la división de los tipos u órdenes a los hechos. Esto le hace especular a Grossmann que en una teoría factualista de las categorías hay una reducción de las variables de entidades de cada uno de los tipos a tan sólo un tipo de variable, a saber, las variables ligadas de los hechos cuantificados. ${ }^{7}$ Hay, a su juicio, sólo un estrato ontológico: los hechos de nuestra experiencia ordinaria. ${ }^{8}$ Todos los tipos de propiedades serán, por tanto, constituyentes ordinarios de hechos.

Como primera consecuencia, esta reducción da lugar a la distinción categorial fundamental de la teoría de las categorías de Grossmann entre hechos, por otro lado, y constituyentes, por otro. Mediante la mencionada variable entidad, Grossmann no define qué categorías de cosas pueden ser constituyentes o no de hechos. Que es la distinción ontológica fundamental de la teoría de las categorías quiere decir que no hay ninguna división del ser que sea menor a partir de la cual se pueda dividir todo lo que existe. La distinción ontológica fundamental de la teoría de las categorías está en relación con su lista de (siete) categorías ${ }^{9}$ en la medida en que sólo las entidades que pertenecen a esta lista son constituyentes de hechos. La segunda consecuencia de la reducción de variables es que la estructura formada por las relaciones entre las categorías de su lista de categorías, expresión que Grossmann recoge sencillamente bajo la expresión "el mundo," ${ }^{10}$ es la representada por las relaciones entre constituyentes de hechos. Para Grossmann, el mundo tiene una estructura porque las relaciones tienen "direcciones", característica fundamental de las relaciones, lo cual significa que son "orden", el orden de los constituyentes de hechos. ${ }^{11}$

1.1. Una dificultad. ¿Está la distinción ontológica fundamental entre individuo y propiedad de la teoría de propiedades realmente presupuesta en todas las clasificaciones científicas? Dado que en algunas inter-

6. El la tercera sección advertiré la importante diferencia que hay, según Grossmann, entre 'estados de cosas' y 'hechos.'

7. CSW: 292. Compárese con la tesis del descenso tipológico de las propiedades a los particulares en G. Bergmann, Realism: A Critique of Brentano and Meinong, Frankfurt, Ontos Verlag, 1967/2004 (de ahora en adelante Realism): 360; y en D. Armstrong, Universals and Scientific Realism: A Theory of Universals, Cambridge, Cambridge University Press, 1978: 137.

8. Compárese con la "tesis del elementalismo" en H. Hochberg, "Elementarism, Independence, and Ontology", en H. Hochberg, Logic, Ontology, and Language, München, Philosophia Verlag, 1984.

9. Por ejemplo, OR: 177.

10. $E W: 86$.

11. Con ello, Grossmann sigue, aunque de manera novedosa, la concepción de G. Bergmann, Realism: 3 y ss.

Thémata. Revista de Filosofía $\mathrm{N}^{\circ} 56$ (2017) pp.: 213-234. 
pretaciones de mecánica cuántica no hay individuos ni propiedades, no es verdad que dicha distinción entre individuo y propiedad esté presupuesta por todas las clasificaciones científicas. En contraste con la idea de Grossmann, yo defiendo que la distinción ontológica fundamental presupuesta por todas las ciencias es la distinción entre 'hecho' y 'constituyente.' ${ }^{12}$

\section{La reducción ontológica}

¿Qué significa tratar de propiedades? Esta es, como Grossmann afirma, "la cuestión de la reducción ontológica"13, en este caso, de las propiedades. Lo que resulta de esta "cuestión" son, según él, siete tesis epistemológicas sobre cómo analizar ontológicamente las propiedades, a saber, (i) la tesis del realismo radical, (ii) la tesis de la equivalencia, (iii) la tesis del empirismo radical, (iv) la tesis del conocimiento directo, (v) la tesis de la intencionalidad, (vi) la tesis del atomismo semántico, y (vii) la tesis del atomismo lógico. El método de análisis que resulta como un todo de las siete tesis epistemológicas es denominado por Grossmann "análisis ontológico".

2.1. La tesis del realismo radical. Esta tesis caracteriza el realismo ontológico de Grossmann, que es una de las dos caras de su filosofía autodenominada por él mismo "realismo empírico". ${ }^{14}$ Para Grossmann, no es una tarea de la ontología decidir si existen o no las propiedades. ${ }^{15}$ No existe, para él, razón por la cual cuestionar las fuentes epistemológicas de los diferentes tipos de propiedades, en particular, el sentido común con respecto a las propiedades ordinarias. Aunque Grossmann acepta la existencia de las propiedades de tipo científico tales como las que descubre el físico, su tesis epistemológica tiene que ver precisamente con aquellos atributos de los individuos y de las diferentes propiedades categoriales que nuestra experiencia cotidiana nos da a conocer, como "ser verde oliva" o "ser una propiedad." Pues todas son en última instancia propiedades ordinarias como resultado de la reducción de variables.

2.2. La tesis de la equivalencia. Una dificultad podría surgir en relación a un posible conflicto entre diferentes fuentes epistemológicas en

12. Para más detalles, Cumpa 2017.

13. R. Grossmann, “A Legacy: Gustav Bergmann”. En Cumpa J. \& Tegtmeier, E. (eds.), Phenomenological Realism Versus Scientific Realism: David M. Armstrong-Reinhardt Grossmann Metaphysical Correspondence, Ontos Verlag, 2009, p. 29. De ahora en adelante, PRusSR.

14. R. Grossmann, The Fourth Way: A Theory of Knowledge, Indiana University Press, 1990, p. vii. De ahora en adelante, $F W$.

15. CSW: 10.

Thémata. Revista de Filosofía $\mathrm{N}^{\circ} 56$ (2017) pp.: 213-234. 
torno a las propiedades. Por ejemplo, mientras el sentido común podría decir que una cierta manzana es coloreda, la física de partículas podría objetar que, puesto que las partes fundamentales no son coloreadas, la manzana no tienen color. Grossmann ha llamado a este argumento, "el argumento fisicalista." La tesis de la equivalencia es la solución de Grossmann al conflicto mencionado entre fuentes epistemológicas. Mediante esta tesis, se introduce una conexión legal entre propiedades ordinarias y científicas. Por ejemplo, la manzana es verde si y sólo si hay una cierta estructura de átomos en tales o cuales estados.

2.3. La tesis del empirismo radical. El empirismo radical es la tesis que funda el realismo epistemológico de Grossmann. También es la otra cara de su llamado "realismo empírico." Según esta tesis, el conocimiento de todas las entidades del mundo externo, tales como individuos, propiedades, relaciones, números, clases, etc., dependen enteramente de la percepción. No hay facultades especial de conocimiento como el 'entendimiento' de Descartes o la 'intuición eidética' de Husserl. ${ }^{16}$

2.4. La tesis del conocimiento directo. La primera característica del realismo epistemológico del sentido común de Grossmann es el llamado "principio de conocimiento directo". Según este principio, el conocimiento del mundo externo, en particular, el de las propiedades es "directo" o no inferido. ${ }^{17}$ En este punto, Grossmann elabora una distinción entre dos tipos de "conocimiento directo", acompañada de una distinción entre dos fuentes de conocimiento o de acto mental, a saber, percepción y experiencia. Ambos actos son actos de conocimiento directo, pero estos difieren en aquello a lo que se dirigen. Para Grossmann, el realismo epistemológico debe adoptar una "versión realista" del principio de conocimiento directo, por la cual son los actos mentales de percepción los que nos dan a conocer las propiedades del mundo externo. Según la característica de exclusión que hay entre actos mentales de percepción y de experiencia, es necesario distinguir, para Grossmann, entre "objetos perceptivos" y "objetos fenoménicos". La percepción es de objetos perceptivos; la experiencia es de objetos fenoménicos.

2.5. La tesis de la intencionalidad. Esta tesis se sigue de la adopción de la versión realista del principio de conocimiento directo. Según esta otra característica del realismo epistemológico de Grossmann, los indefinibles o simples actos mentales de percepción son intencionales. Esto quiere decir que tienen la característica de dirigirse hacia un objeto o de

16. $F W$ : vii.

17. R. Grossmann, "Bergmann's Ontologies and the Principle of Acquaintance". En Gram M. S. and Klemke E. D. (eds.), The Ontological Turn: Studies in the Philosophy of Gustav Bergmann, Iowa City, University of Iowa Press, 1974, p. 89.

Thémata. Revista de Filosofía N56 (2017) pp.: 213-234. 
ser directos. ${ }^{18}$ Pero, ¿qué es, Grossmann pregunta, 'dirigirse hacia un objeto"? Esta no es sino su fenomenología o "teoría de las intenciones". ${ }^{19}$ Es por la existencia de una relación, el "nexo intencional", que los actos mentales pueden tender a un objeto. ${ }^{20} \mathrm{El}$ tipo de acto mental, y así sus direcciones, internas o externas, del "nexo intencional", se distinguen entre sí, según Grossmann, mediante dos propiedades que tienen todos los actos mentales, la clase y el contenido. Por la 'clase,' los actos mentales se distinguen cualitativamente entre sí, por ejemplo, un acto mental de ver de uno de juzgar; por el 'contenido,' los actos mentales se distinguen entre sí por los objetos que tienen. Esto podemos llegar a saberlo, según Grossmann, mediante lo que llama "introspección". ${ }^{21}$ En línea con la diferencia entre objetos fenoménicos y perceptivos, Grossmann explica la diferencia experimentada entre actos mentales según el contenido. ${ }^{22}$

2.6. La tesis del atomismo semántico. La terminología de "objetos perceptivos" conduce a Grossmann a plantearse dos preguntas: ¿Por qué los actos mentales de percepción tienden a objetos perceptivos? y ¿qué es, pues, un objeto perceptivo? Las respuestas a estas dos preguntas constituyen la tercera y la cuarta característica del realismo epistemológico de Grossmann. A saber, la "tesis del atomismo semántico" ${ }^{23}$ y la "tesis del atomismo lógico". ${ }^{24}$ Según la tesis enunciada en este parágrafo, un acto mental de percepción tiende a objetos perceptivos más bien que a entidades aisladas o descontextualizadas porque es proposicional. Lo que es proposicional son "los contenidos" de los actos mentales de percepción. Según Grossmann, todos los contenidos de los actos mentales de percepción, incluidos los aparentemente "simples" como conceptos sobre un individuo o una propiedad, son de tipo proposicional (lingüísticamente representados por cláusulas como 'que $p$ ')..$^{25}$

2.7. La tesis del atomismo lógico. Como respuesta a la segunda pregunta del parágrafo anterior, de acuerdo con parte de la tesis del ato-

18. R. Grossmann, "Materialism and the New Folk Philosophy". En Cumpa, J. (ed.), Studies in the Ontology of Reinhardt Grossmann, Ontos Verlag, 2010, pp. 269-270.

19. R. Grossmann, "Introduction". En K. Twardowski, On the Content and the Object of Presentations, Martinus Nijhoff, 1977, p. IX.

20. $F W: 51-52$.

21. R. Grossmann, The Structure of Mind, Madison, Northwestern University Press, 1965, p. 5 y ss. De ahora en adelante, $S M$.

22. SM: 218.

23. R. Grossmann, Phenomenology \& Existentialism: An Introduction, London, Rouledge, 1984, pp. 108-109. De ahora en adelante, $P E$.

24. OR: 163 , nota al pie.

25. SM: 112.

Thémata. Revista de Filosofía $\mathrm{N}^{\circ} 56$ (2017) pp.: 213-234. 
mismo lógico, los "objetos perceptivos" son hechos, entidades en contextos. Esto hace de la categoría dehecho la categoría central de la ontología de Grossmann. ${ }^{26}$ Pero, ¿qué hay de las propiedades?, ¿no pueden ser conocidas directamente? Para Grossmann, pueden ser tan directamente conocidas como los hechos, por la razón de que cada acto mental de percepción tiene más de un objeto. Tiene objetos primarios y secundarios. ${ }^{27}$ Ahora bien, "primario" y "secundario" no son expresiones que deban ser entendidas expresando un orden en la inmediatez de la percepción, sino más sólo el número de objetos percibidos. Según la segunda parte de la tesis, los constituyentes de hechos, en nuestro caso particular, las propiedades, son entidades que son simples. ${ }^{28}$

\section{El análisis ontológico: qué es y qué no es}

En un parágrafo anterior Grossmann mostró que no es una tarea de la ontología decidir qué propiedades existen. Ahora Grossmann mostrará, en primer lugar, que tampoco es una tarea tratar de propiedades que no existen, son posibles o son no-existentes. Una vez mostrado esto, Grossmann nos presentará el análisis ontológico para ver cómo difiere de otros tipos de análisis de las propiedades. Según Grossmann, la condición epistemológica a tener en cuenta en el resultado del análisis ontológico es que se consideren sólo los actos mentales de percepción verídica. La razón es que son los únicos actos mentales que tienen "objetos perceptivos" o "estados de cosas" que existen, que son hechos, a diferencia de lo que ocurre, por ejemplo, con los objetos perceptivos de actos mentales de percepción no verídicos tales como los actos mentales de percepción alucinatoria. Esta es, naturalmente, la razón por la que Grossmann tiene que enfrentarse al problema de las propiedades posibles o no-existentes. A juicio de Grossmann, la condición ontológica a tener en cuenta en el resultado del análisis ontológico es que los objetos de los actos mentales de percepción sean estados de cosas existentes o hechos. Grossmann no incluye, pues, los constituyentes posibles o que no existen como susceptibles de posible análisis ontológico, por la simple razón de que no hay nada en ellos que sea susceptible del

26. OR: 11 y nota al pie de página.

27. CSW: 406.

28. $C S W: 6$.

Thémata. Revista de Filosofía N56 (2017) pp.: 213-234. 
análisis. Grossmann señala que el Meinong de la "Teoría de los objetos" es el principal oponente de su concepción. ${ }^{29}$

Grossmann rechaza la posibilidad de que individuos, propiedades y relaciones puedan ser constituyentes posibles o no-existentes de hechos. Comienza distinguiendo entre "cosas no-existentes" y "estados de cosas no-existentes", que es una distinción entre "individuos", "atributos: propiedades y relaciones" y "estados de cosas", todos los cuales son entidades no-existentes. ${ }^{30}$ Examinando tanto supuestos hechos alegados por Meinong como su discusión por parte del Russell de "La teoría de Meinong de complejos y asunciones" ${ }^{1}$, Grossmann toma dos argumentos del último para rechazar la tesis de Meinong de que individuos posibles o no-existentes puedan ser constituyentes de hechos. El primero de ellos es que el alegado individuo posible por parte de Meinong, "la montaña dorada", no es constituyente de ningún hecho, porque no es un constituyente de ningún hecho ni sobre su existencia ni, en particular, sobre su no-existencia. El segundo es un argumento de Russell en contra de la réplica de Meinong al primer argumento de Russell. Según la réplica de Meinong, el hecho de que ningún existente es un fantasma tiene un individuo posible o no-existente, un fantasma, como constituyente, por la razón de que al pensar en ello se piensa sobre una entidad existente, independientemente de si es una entidad vaga o más bien de un tipo determinado. Pero Grossmann, siguiendo de nuevo a Russell, defiende que pensar indiscriminadamente sobre cualquier cosa no implica 'cuantificar' en absoluto. Lo cuantificado, según Grossmann, en el caso de los objetos no-existentes o posibles, no es la "variable entidad o existencia" que encontramos en los hechos cuantificados, sino más bien lo que llama la "variable objeto", de estados de cosas posibles o no-existentes (cuantificados). Pero, para Grossmann, tampoco hay propiedades y relaciones posibles o no-existentes. En lo esencial, Grossmann rechaza la supuesta existencia de propiedades y relaciones posibles o no-existentes por tres razones. La primera es que parece depender del capricho de nuestra imaginación afirmar que el estado de cosas contiene una propiedad mejor que otra como constituyente. La segunda es que del hecho de que estas propiedades posibles o no-existentes se entiendan como complejos de propiedades, y que tales propiedades se entiendan como constituyentes necesarios de esos complejos, no se puede inferir la existencia de tales complejos de propiedades. Esto sería el caso, para Grossmann, si y sólo si existiera la cosa a la que se le atribuyen semejantes

29. A. Meinong, "Gegenstandstheorie”. En A. Meinong, Gesammelten Abhandlungen. Band. II, Leipzig, 1903/1913.

30. CSW: 194.

31. B. Russell, "Meinong's Theory of Complexes and Assumptions". En Mind, Vol. XIII, 1904.

Thémata. Revista de Filosofía $\mathrm{N}^{\circ} 56$ (2017) pp.: 213-234. 
propiedades. La tercera razón es que, si esto es así, las relaciones posibles o no-existentes entre individuos, y entre individuos y propiedades desaparecen.$^{32} \mathrm{El}$ análisis ontológico es, según Grossmann, un método de análisis empírico con dos fases y metas diferentes. Estas fases, como él insiste, son siempre susceptibles de revisión. Es en la primera fase del análisis de los tipos de hechos, donde encontramos individuos y atributos ordinarios existentes como objetos primarios y secundarios de actos de percepción verídica. Es en el segundo nivel de análisis ontológico en que se enumeran las categorías. Para Grossmann, tal enumeración se distingue del "análisis de clases." ${ }^{33}$ Ahora bien, Grossmann piensa que el análisis ontológico de las propiedades no está libre de alteración por otros tipos de análisis, en particular, el lingüístico, el fenoménico y el espacial. Para Grossmann, el análisis lingüístico, fenoménico y el espacial no forman parte del análisis ontológico.

3.1.1. El análisis lingüístico. ¿Qué expresiones lingüísticas representan atributos ordinarios y qué expresiones lingüísticas no lo hacen? Grossmann no considera que un enunciado bien formado sobre atributos ordinarios deba "representar" un hecho correspondiente con una cierta propiedad o relación. Denomina él a esta tesis "principio de abstracción de propiedades" y señala al Russell de Los principios de las matemáticas como el mayor proponente de ello. ${ }^{34}$ Grossmann pone en cuestión tanto "la representabilidad" como "lo representado" del principio. En cuanto a lo primero, Grossmann argumenta que los atributos ordinarios "imaginados" son atributos ordinarios convenidos lingüísticamente, meras definiciones contextuales, "la ruina de la ontología", escribe él..$^{35}$ Para Grossmann, las expresiones predicativas pueden tener una relación de representación con algo si y sólo si se basan en actos mentales de significar que comparten el contenido de un acto mental previo de percepción verídica.$^{36}$ Con respecto a lo segundo, Grossmann considera cuestionable la creencia que apoya la ya criticada representabilidad: el "dogma de las propiedades complejas". En este punto, Grossmann se pregunta qué expresión compleja podría representar un atributo complejo. Puesto que no hay semejante atributo del que se pueda tener conocimiento directo, la conclusión de Grossmann es que el

32. CSW: 409-412.

33. CSW: 6-9.

34. B. Russell, The Principles of Mathematics, Norton \& Company, 1964.

35. $C S W: 12$ y 16.

36. CSW: 203.

Thémata. Revista de Filosofía N56 (2017) pp.: 213-234. 
análisis lingüístico de expresiones predicativas no es básico para obtener conocimiento de los atributos. ${ }^{37}$

3.1.2. El análisis fenoménico. Grossmann denomina "argumento de la relatividad del sentir" al argumento que conduce a interpretar el análisis ontológico como análisis fenoménico. ${ }^{38}$ Los proponentes son, a su parecer, el Russell de Los problemas de la filosofía ${ }^{39}$ y el Husserl de Ideas $1 .^{40}$ De este argumento, se siguen, según Grossmann, dos conclusiones. La primera es que el principio de conocimiento directo del análisis ontológico de los atributos ordinarios es "fenomenalista", es decir, "por inferencia" de nuestras impresiones sensibles. La segunda es que, puesto que hay diferentes inferencias de nuestras impresiones sensibles desde diferentes "aspectos" de las propiedades, no podemos saber cuál es la verdadera propiedad a la que tiende el objeto del acto mental verídico de percepción. Grossmann rechaza la primera conclusión porque confunde percibir objetos perceptivos o atributos ordinarios con experimentar sensaciones producidas por los primeros. Grossmann rechaza la segunda porque el supuesto de que el conocimiento por descripción de los atributos ordinarios no proporciona conocimiento es falso. Pues aunque las propiedades no son aspectos porque no es necesario inferirlas de las propiedades de las sensaciones, podemos conocerlas a través de la descripción de sus aspectos. ${ }^{41}$

3.1.3. El análisis espacial. "Es obvio" - escribe Grossmann- "que el análisis espacial no debe confundirse con el análisis ontológico". ${ }^{42}$ En particular, declara que no debe confundirse con dos tipos de análisis espacial. El primero afecta al primer nivel de análisis ontológico. Este tipo de análisis espacial tiene a su vez dos variantes. Grossmann sugiere que ambas resultan de la confusión del análisis ontológico con el análisis fenoménico. En la primera variante, el análisis ontológico se confunde con el análisis de los atributos de las partículas elementales; en la segunda, con el análisis de los atributos deducidos de los sistemas nomológico-deductivos. Ambas comparten, según Grossmann, la tesis de que los atributos ordinarios son "reducibles" en un sentido. Estos dos sentidos tienen que ver con cada una de las variantes. Los proponentes de estos tipos de análisis son, respecti-

37. R. Grossmann, "Perceptual Objects, Elementary Particles, and Emergent Properties". En Castañeda, H. N. (ed.), Action, Knowledge, and Reality: Critical Studies in Honour of Wilfrid Sellars, Bobbs-Merrill Company, 1975, p. 138.

38. $F W: 74$.

39. B. Russell, The Problems of Philosophy, Home University Library, 1912).

40. E. Husserl, Ideas Pertaining to a Pure Phenomenology and to a Phenomenological Philosophy, Collier Books, 1913/1962.

41. $F W: 74-76$ y $132-133$.

42. OR: 162 .

Thémata. Revista de Filosofía Nº56 (2017) pp.: 213-234. 
vamente, el Wilfrid Sellars de Ciencia, percepción y realidad ${ }^{43}$ y el Hempel y Oppenheim de "Estudios de la lógica de la explicación científica" ${ }^{44}$ Además, Grossmann atribuye al David Armstrong del artículo "La ontología de Reinhardt Grossmann" ${ }^{45}$ ser proponente de un subtipo de análisis espacial perteneciente al segundo sentido de reducción. Grossmann ha incluido los tres análisis en lo que ha llamado "el argumento fisicalista" . ${ }^{46}$ Según Grossmann, el análisis espacial de Sellars, denominado por él "principio de reducción", conduce a la conclusión de que un atributo ordinario de una cosa individual, por ejemplo, 'verde', es reducible a atributos ordinarios de partes espaciales últimas de la cosa individual, es decir, a los de las partículas elementales de la física de partículas. Para Grossmann, mientras que la tesis de que las cosas individuales son sistemas de partículas elementales y la de que las partículas elementales de estos sistemas no tienen atributos como el color, le parecen inobjetables, no le parece que se siga que las cosas individuales como sistemas no tengan atributos ordinarios. Esto es de acuerdo con su anteriormente mencionado "realismo radical". Tomando la distinción de Sellars entre "imagen manifiesta" e "imagen científica", Grossmann afirma que lo reducible al sistema de partículas elementales de la imagen científica es la cosa individual, no los atributos que tiene de la imagen manifiesta. ${ }^{47}$

El sentido de "reducción" del análisis espacial propuesto por Hempel y Oppenheim es diferente del de Sellars. Esta variante de análisis espacial consiste en la idea de que un atributo ordinario es reducible a otros atributos ordinarios si su ocurrencia se puede deducir de ciertas leyes. Grossmann no objeta nada, puesto que considera que todos los atributos ordinarios de las cosas individuales son perfectamente deducibles de las leyes de la física. ${ }^{48}$ Como en el caso de Sellars, también Grossmann trata de conciliar este sentido de análisis espacial con el sentido común. Pero mientras que Grossmann acepta este sentido de reducción de los atributos ordinarios que proponen Hempel y Oppenheim, sin embargo, rechaza la interpretación identificadora de tipo Feigliano que de este sentido de reducibilidad hace David M. Armstrong. ${ }^{49}$ Según Armstrong, las conexiones

43. W. Sellars, Science, Perception and Reality, Routledge and Kegan Paul, 1963).

44. C. G. Hempel y P. Oppenheim, "Studies in the Logic of Explanation". In H. Feigl and M. Brodbeck (eds.), Readings in the Philosophy of Science, Appleton-Century-Crofts, 1948/1953. 45. D. M. Armstrong, "Reinhardt Grossmann's Ontology”. En J. Cumpa \& E. Tegtmeier (ed.), PRusSR.

46. $F W: 58$ y ss.

47. $F W: 61$.

48. $F W: 61$.

49. D. M. Armstrong, "Reinhardt Grossmann's Ontology”. En J. Cumpa \& E. Tegtmeier (ed.),

Thémata. Revista de Filosofía $\mathrm{N}^{\circ} 56$ (2017) pp.: 213-234. 
legales entre propiedades que establece el método nomológico-deductivo que Grossmann acepta tiene como consecuencia la introducción de leyes sospechosas que no encajan con el sistema de leyes establecidas. Por esta razón, Armstrong interpreta que las conexiones legales establecen identidades más bien que las equivalencias de Grossmann. El argumento de Grossmann en contra de la interpretación de Armstrong es que si fuera verdad que la relación fuera una identidad, entonces no sería posible distinguir los términos de la relación, digamos, los colores y los estados de las estructuras atómicas. ${ }^{50}$

El segundo tipo de análisis espacial afecta al segundo nivel de análisis ontológico de las propiedades en la forma de que no es posible llegar a descubrir los simples ontológicos, átomos o categorías que Grossmann mencionó en su "tesis del atomismo lógico". Puesto que se confunde el análisis ontológico, en particular, su segundo nivel, con el análisis espacial, lo único que cabe esperar es una división espacial infinita de los atributos ordinarios, por la cual será imposible alcanzar su propiedad categorial o categoría. ${ }^{51}$ Esta confusión está representada, a juicio de Grossmann, por el Wittgenstein de Los cuadernos de notas $^{52}$ y de Las investigaciones filosóficas. ${ }^{53}$ La confusión de Wittgenstein, según Grossmann, tiene dos fases. En la primera, la de Los cuadernos de notas, Wittgenstein afirma que la complejidad y la simplicidad categorial (lógica) son la complejidad y la simplicidad espaciales. Identificar los complejos lógicos con los complejos espaciales es la razón de la confusión del análisis ontológico de hechos con el análisis espacial de cosas individuales. Identificar los simples lógicos o categoriales con los "simples espaciales" es, a juicio de Grossmann, lo que conduce a Wittgenstein en Las investigaciones filosóficas a rechazar la idea, propuesta previamente en el Tractatus Logico-Philosophicus, de que existen simples lógicos o categorías. El primer argumento de Grossmann para bloquear la primera conclusión de Wittgenstein es destacar que el análisis espacial no puede conducir al descubrimiento de otras categorías diferente de la de individuo. El segundo argumento de Grossmann para bloquear la segunda conclusión de Wittgenstein es mostrar que la división espacial infinita de una propiedad no elimina la categoría de propiedad. ${ }^{54}$

3.1.3.1. Una dificultad: ¿Es sostenible la interpretación de la individualidad de Grossmann en su solución del 'argumento fisicalista,' de

PRvsSR.

50. FW: 62-63.

51. OR: 163, nota al pie y p. 167.

52. L. Wittgenstein, Notebooks: 1914-1916, Blackwell, 1961.

53. L. Wittgenstein, Philosophical Investigations, Oxford University Press, 1954.

54. RF: 98.

Thémata. Revista de Filosofía Nº56 (2017) pp.: 213-234. 
acuerdo con la cual los individuos son idénticos con sistemas de partículas? Teniendo en cuenta las múltiples interpretaciones de la mecánica cuántica en las que simplemente no hay ni individuos, ni en algunas de ellas si quiera partículas elementales, y también teniendo en cuenta el fenómeno llamado "entrelazamiento cuántico," por el que los sistemas cuánticos no se reducen a sus partes, la solución de Grossmann parece ser meramente dependiente de la interpretación clásica de la individualidad. Con el fin de evitar consecuencias de este tipo, yo defiendo un planteamiento factualista de la composición de los objetos ordinarios, que es totalmente compatible con diferentes modelos de la física..$^{55}$

\section{Las tareas de la teoría de propiedades}

Una vez mostradas cuáles no son las tareas de la ontología, mostrando qué no es el análisis ontológico, Grossmann anuncia las dos tareas de la teoría de propiedades. Tales tareas consisten en la solución de tres problemas del segundo nivel del análisis ontológico que titula "la cuestión realismo-nominalismo:" (1) ¿Qué es una propiedad o a qué categoría pertenece? y (3) ¿cuál es la relación entre y las propiedades y las cosas que las tienen? ${ }^{56}$ Según Grossmann, las dos tareas de la teoría de propiedades se aplican a los tres tipos de propiedades. Y propone una solución a cada una de ellas a la luz de nueve características que atribuye a las propiedades: (i) simplicidad, (ii) identidad cualitativa, (iii) inespacialidad, (iv) atemporalidad, (v) dependencia, (vi) externalidad, (vii) accidentalidad, (viii) contingencia, y (ix) existencia unívoca.

4.1. Simplicidad. Por "propiedad simple" o "simplicidad de una propiedad", Grossmann entiende propiedad "fenomenológicamente" simple. Ahora bien, esto no quiere decir "aparentemente simple", sino "ontológicamente simple." Grossmann escribe en una carta a David Armstrong, "¿Qué tendríamos que descubrir, por ejemplo, para que (...) [una propiedad] no sea simple? Lo único que pienso que podría descubrir es, me parece, que esta propiedad está conectada con otras propiedades (mediante "si y sólo si" o "si, entonces"), pero no que consista de ellas". ${ }^{57}$ Si la simplicidad fenomenológica no fuera el criterio de simplicidad de las propiedades, entonces Grossmann afirma que "no podríamos distinguir entre conexión le-

55. Para detalles sobre mi solución, véase Cumpa 2017.

56. CSW: 3 y 12.

57. R. Grossmann, "On the Simplicity of Universals". En Cumpa, J. \& Tegtmeier, E. (eds.), PRusSR: 27. Compárese con las observaciones de H. Hochberg, "Review of Phenomenological Realism Versus Scientific Realism”. En Dialectica, Vol. 64, N 3, 2010, pp. 447-451.

Thémata. Revista de Filosofía N56 (2017) pp.: 213-234. 
gal entre propiedades, por un lado, y complejidad de propiedades, por otro. Este criterio [simplicidad fenomenológica] es el único criterio que conozco que nos permite hacer esta distinción. ${ }^{58}$ Grossmann considera que es necesario poner en cuestión un supuesto del tipo de análisis espacial del "consistir" propuesto por Armstrong, a saber, que hay propiedades más importantes que otras. Las propiedades más importantes son las que descubre la ciencia. De este supuesto depende, a juicio de Grossmann, la elección de "la tesis de la equivalencia" o "la tesis de la identidad". En este sentido, si las propiedades se reducen a complejos de propiedades más simples no es nada más que una elección de la importancia de uno de los dos términos de la relación en cuestión. ${ }^{59}$

4.2. Identidad cualitativa. Grossmann considera que no es razonable en absoluto pedir un criterio de identidad para las entidades simples como las propiedades. Sin embargo, propone uno de acuerdo con la simplicidad de las propiedades, a saber, el "principio de la identidad de los indiscernibles". ${ }^{60}$ Según el principio, dos propiedades son idénticas si y sólo si comparten todos sus atributos. Con el fin de apoyar el principio en cuestión como condición de identidad de las propiedades, Grossmann sugiere que su no aceptación conduce a un regreso infinito de la identidad de la propiedad. ${ }^{61}$ Tomando partido de sus argumentos en contra de las propiedades complejas, propone una segunda condición de identidad para propiedades simples: dos propiedades son idénticas si y sólo si determinan la misma clase. ${ }^{62}$ Grossmann llama "realista" a quien acepta que las propiedades son identidades cualitativas o también o universales. Denomina "nominalista" a quien afirma la tesis opuesta al realismo, a saber, que las propiedades no son identidades cualitativas o universales, sino más bien semejanzas o particulares. Hay dos argumentos que Grossmann considera "naturalistas", uno ontológico y otro epistemológico, que apoyan la tesis del nominalismo sobre las propiedades. Grossmann los llama respectivamente, el "axioma de localización" 63 y el "dogma de la localización". ${ }^{64}$ Por el primer argumento, todo lo que existe está localizado en un lugar del espacio y en un momento del tiempo. Por el segundo argumento, todos los

58. R. Grossmann, “Objections”. En Cumpa, J. \& Tegtmeier, E. (eds.), PRvsSR: 26.

59.8 CSW: 139-141.

60. CSW: 374; énfasis mío.

61. EW: 24.

62. CSW: 282 .

63. $E W: 30$.

64. R. Grossmann, "Sensory Intuition and the Dogma of Localization”. En Allaire, B. E. (ed.), Essays in Ontology, The Hage, Martinus Nijhoff, 1963, pp. 50. De ahora en adelante, $D L$.

Thémata. Revista de Filosofía Nº56 (2017) pp.: 213-234. 
objetos de la percepción están localizados en el espacio y en un momento del tiempo.

4.3. Inespacialidad. Que "dónde," i.e., las relaciones espaciales no son atributos de las propiedades es la afirmación principal de Grossmann. Para ello, rechaza dos argumentos a favor de la localización espacial de las propiedades. Uno de ellos es aducido por el Nicholas Wolterstorff de "Las cualidades"65, y el otro, tanto por el Edmund Husserl de Las investigaciones lógicas ${ }^{66}$ así como también por el George Stout de "La naturaleza de los universales y las proposiciones." 67 El primer argumento de Grossmann es en contra del "axioma de localización". Él corrige un argumento de Wolterstorff, según el cual las propiedades están en el espacio porque a la pregunta “¿dónde están las propiedades?” se puede responder por indicación. Grossmann piensa que la atribución de estas relaciones espaciales a las propiedades carece de sentido. Lo que la atribución del "dónde" parece indicar más bien es, para Grossmann, que la propiedad está localizada sólo en función de la localización espacial de la cosa, la bola de billar, que la tiene. ${ }^{68}$ Esto conduce a Grossmann a la tesis de que las propiedades no son partes espaciales de los individuos. Por tanto, Grossmann concluye que a diferencia de los individuos que las tienen, las propiedades no están en el espacio, son inespaciales. Pero, ¿qué significa entonces "estar localizado" para las propiedades? Según Grossmann, hay dos tipos categorialmente diferentes de estar localizado. En primer lugar, una entidad está localizada si y sólo si ejemplifica relaciones espaciales y/o temporales. Y en segundo lugar, una entidad está localizada si y sólo si es ejemplificada por un particular. ${ }^{69}$

El segundo argumento de Grossmann es en contra del "dogma de la localización" que han propuesto Husserl y Stout. Según este argumento epistemológico, la percepción sensible sólo nos puede dar a conocer entidades localizadas en el espacio. El principio de la identidad de los indiscernibles conduce a Grossmann a pensar que no hay ninguna razón para aceptar el dogma. ${ }^{70}$

4.4. Atemporalidad. Que "cuándo," es decir, las relaciones temporales no son atributos de las propiedades es la tesis central de Grossmann

65. N. Wolterstorff, "Qualities". En Landesman, C. (ed.), The Problem of Universals, Basic books, 1971, pp. 200- 212.

66. E. Husserl, Logical Investigations, 2 Vols., Humanity Press, 1970, Vol. I.

67. G. F. Stout, "The Nature of Universals and Propositions". En Proceedings of the British Academy, 1921-1922, Vol. X.

68. EW: 44.

69. DL: 57.

70. CSW: 109-112.

Thémata. Revista de Filosofía N56 (2017) pp.: 213-234. 
en este contexto. Ahora el oponente de Grossmann es el Alexius Meinong de Los estudios sobre Hume $I .{ }^{71}$ Según el argumento de Meinong, las propiedades son temporales, existen en el tiempo, porque cuando la cosa que las tiene deja de existir, sus propiedades también dejan de existir en ese mismo momento. El argumento de Grossmann contra la concepción de Meinong consiste en mostrar que el argumento de Meinong depende del argumento anterior a favor del carácter espacial de las propiedades. Sólo si las propiedades son "partes espaciales" de los individuos, se puede afirmar que son temporales, en este caso, que cuando cesa de existir un individuo también dejan de existir sus propiedades. ${ }^{72}$

4.5. Dependencia. Para Grossmann, el "nexo de ejemplificación", la relación entre individuos y propiedades, es central para lograr un análisis ontológico satisfactorio de las propiedades como universales ${ }^{73}$ En primer lugar, Grossmann defiende que el nexo no es una relación espacial parte-todo. Según esta característica, es más bien una relación abstracta. En segundo lugar, Grossmann sostiene que el nexo de ejemplificación es no-simétrico. ${ }^{74}$ Esto significa que los individuos tienen propiedades pero no viceversa, de modo que, de acuerdo con la característica anterior, es una relación heterogénea o que cruza categorías, es decir, un "enlace" (tie) entre categorías diferentes. En tercer lugar, Grossmann insiste en que el nexo no tiene instancias, es decir, que es universal. ${ }^{75}$ Esto quiere decir que puede estar en individuos diferentes al mismo tiempo. Pero el hecho de que el nexo de ejemplificación no sea una relación espacial parte-todo, no quiere decir, a juicio de Grossmann, que las propiedades puedan existir sin estar ejemplificadas en al menos un individuo. Como afirmó, el nexo es una ley categorial de mutua "dependencia" entre individuos y propiedades por la que toda propiedad está ejemplificada por al menos una entidad, y toda entidad ejemplifica al menos una propiedad. ${ }^{76}$

4.6. Externalidad. Ahora bien, la mencionada "dependencia" del nexo no implica, según Grossmann, que el nexo de ejemplificación sea una relación esencial o necesaria entre particulares y universales. Para él, es una relación externa, no-interna, puesto que no se basa en la naturaleza

71. A. Meinong, Hume Studien I, en Haller, R. (ed.) Gesamtausgabe, 7 Vols., Verlaganstalt, Vol. I, 1968-1978.

72. R. Grossmann, R. Meinong, London, Routledge, 1974, pp. 6-7.

73. CSW: 118.

74. R. Grossmann, "The Nature of Universals". En Cumpa, J. \& Tegtmeier, E. (eds.), PRvsSR: 34.

75. CSW: 122.

76. Compárese con el "principio de ejemplificación” en G. Bergmann, Logic and Reality, Madison, University of Wisconsin Press, 1964, p. 245; y en D. M. Armstrong, Universals and Scientific Realism: Nominalism and Realism, Cambridge, Cambridge University Press, 1978, p. 113.

Thémata. Revista de Filosofía №56 (2017) pp.: 213-234. 
de los términos que relaciona. ${ }^{77}$ Esta externalidad del nexo de ejemplificación - una tesis central de la "Escuela de Iowa" de Gustav Bergmann a la que Grossmann pertenece- muestra la independencia que existe entre las dos categorías de los particulares y los universales. Este tipo de independencia es por lo que entra en escena el nexo de ejemplificación. Según Grossmann, la "dependencia" expresada por el nexo es el único tipo de dependencia ontológica entre particulares y propiedades. ${ }^{78}$ Esta es, natutralmente, una característica del nexo, no de las propiedades.

4.7. Accidentalidad. Pero, ¿qué hay de la distinción entre propiedades esenciales y accidentales: no funda, entonces, un tipo de dependencia ontológica diferente del expresado por el nexo de ejemplificación? Si no hay propiedades individuales, sino sólo individuos, tal que no hay las clásicamente llamadas "sustancias segundas" dividuo, a Grossmann le parece que no se puede establecer una distinción entre propiedades esenciales y accidentales. ${ }^{80}$ Esto significa que todas las propiedades son accidentales y que todas se relacionan de la misma manera externa con los individuos. ${ }^{81}$ Siguiendo a Locke, Grossmann piensa que la distinción sólo se puede establecer de manera "relativa" o "no absoluta", tomando las propiedades esenciales como "esencias nominales". ${ }^{22}$ Según esto, una propiedad, digamos, ser racional, es esencial si es relativa a otra, digamos, ser humano, que un individuo, por ejemplo, Platón, ejemplifica. Esto significa, a juicio de Grossmann, que las propiedades esenciales son propiedades complejas cuya relación entre ellas es una relación de partetodo de "consistir en" de tipo analítico. Por ejemplo, ser humano es una propiedad compleja que tiene como una de sus partes la propiedad de ser racional. De esta manera, decir que Platón es esencialmente racional es decir que Platón es racional y que es racional se sigue por medio de una ley del hecho de que es un ser humano. Para Grossmann, esto no parecería requerir la introducción de tipos nuevos de propiedad y ejemplificación. ${ }^{83}$ Según el, las propiedades son, por tanto, accidentales.

4.8. Contingencia. Pero una vez más, Grossmann se pregunta: ¿qué hay de la distinción entre propiedades necesarias y contingentes?

77. Por ejemplo, Allaire, B. E., "Existence, Independence and Universals". En Allaire, B. E. et alii (ed.), Essays in Ontology, The Hage, Martinus Nijhoff, 1963.

78. CSW: 60-61 y 118-120.

79. Aristóteles, "Categorías". En Categories and De Interpretatione, Oxford, Clarendon Press, 1963: 1a20-1b6.

80. CSW: 135.

81. CSW: 61.

82. CSW: 136-137.

83. CSW: 139 .

Thémata. Revista de Filosofía N56 (2017) pp.: 213-234. 
¿No funda, pues, esta un tipo de dependencia ontológica diferente del expresado por el nexo de ejemplificación? Las propiedades necesarias son, como las esenciales, relativas a algo y, como tal, tienen que explicarse, según Grossmann, en los mismos términos que las propiedades esenciales, a saber, el nexo de ejemplificación, la conexión legal entre propiedades, y la implicación lógica. Puesto que los particulares no tienen propiedades esenciales más que en términos relativos, Grossmann confiesa sólo ser capaz de dar sentido de la noción de necesidad absoluta (de re) en exactamente los mismos términos. En este sentido, decir que "Platón es necesariamente racional es decir que "Platón es racional y que es racional se sigue por medio de una ley del hecho de que es un ser humano." ${ }^{44}$

Por último, Grossmann se pregunta si el tipo de necesidad entre propiedades llamada "sintética a priori" funda un tipo de dependencia ontológica diferente del de la ejemplificación. Un ejemplo de este tipo de necesidad entre propiedades es: "no hay color sin extensión". Sin embargo, este tipo debería poder, según Grossmann, ser analizado también en términos de ejemplificación, conexión legal entre propiedades, e implicación lógica. Para saberlo, Grossmann plantea cuál es la fuente de la necesidad de lo sintético a priori. A ello responde que "lo que podemos imaginar y lo que no podemos imaginar depende de nuestros órganos sensoriales. Organismos con diferentes percepciones, por ejemplo, podrían imaginar cosas que nosotros no podemos. Esto tendría que recordarnos la frágil naturaleza de este tipo de necesidad" ${ }^{85}$ Según esta concepción de Grossmann, decir que "no hay color sin extensión" es más bien decir lo siguiente: "Platón, en la media de que tiene color, no se puede imaginar sin extensión. Puesto que ejemplifica esta propiedad, es inimaginable que no deba tener la otra propiedad. ${ }^{86}$

4.9. Existencia unívoca. Una consecuencia positiva de la reducción de las variables afecta, según Grossmann, al tipo de existencia que tienen las propiedades. Puesto que la reducción de las variables no permite relacionar la existencia con los tipos de variables de la jerarquía, ${ }^{87}$ Grossmann piensa que ello implica que no hay modos de ser o existir diferentes para cada tipo o categoría. ${ }^{88} \mathrm{De}$ acuerdo con la reducción, todo lo que representan las variables ligadas de los hechos cualificados existe sin ninguna prioridad ontológica. En las palabras del propio Grossmann, lo representado por la variable es "todo: la variable forma una categoría trascenden-

84. $C S W: 141-142$.

85. CSW: 90.

86. $C S W: 142$.

87. CSW: 387-401.

88. CSW: 291-292 у 387-392.

Thémata. Revista de Filosofía $\mathrm{N}^{\circ} 56$ (2017) pp.: 213-234. 
tal." ${ }^{89} \mathrm{El}$ estatus ontológico de las propiedades, por tanto, es unívoco. Es , a su juicio, simplemente existir $\cdot{ }^{90} \mathrm{Si}$ las propiedades son reales o mentales, este no es un problema ontológico, según Grossmann, que pertenezca a la "cuestión realismo-nominalismo," sino más bien un problema de la filosofía de la mente que pertenece a la llamada "cuestión realismo-idealismo."

4.10. Una dificultad: ¿no debiera Grossmann incluir como tareas de la teoría de propiedades las de intentar resolver los problemas ontológicos relacionados con su concepción de la reducción ontológica y el análisis ontológico de las propiedades? Las tareas de resolver estos problemas tan concretos no parecen menos importantes que la de resolver los más abstractos, como acabamos de ver. De hecho, como Grossmann manifiesta en algunas ocasiones, la solución de los primeros problemas repercute en la de los últimos. Por otro lado, tal y como la ciencia se va desarrollando en la actualidad cada vez surgen más desafíos en estos dos contextos sobre las propiedades y una teoría ontológica sobre las propiedades no puede considerarlos no ser tareas indispensables. Quizás el hecho de que Grossmann conceda mayor importancia a las tareas ontológicas de la teoría de propiedades relativas al problema de los universales explica que se le planteen problemas ontológicos tan serios como los mencionados anteriormente en torno a la mecánica cuántica. Es bastante razonable, por tanto, incluir las tareas ontológicas mencionadas de esos dos contextos en la lista de tareas de la teoría de propiedades. En mi opinión, la tarea genuina de la ontología se encuentra en esos dos contextos y no en la búsqueda de categorías o en la categorización de las propiedades como particulares o universales ${ }^{91}$

\section{Bibliografía}

Allaire, B. E., 1963, "Existence, Independence and Universals". En Allaire, B. E. (ed.), Essays in Ontology, The Hage, Martinus Nijhoff.

Aristóteles, 1963, "Categorías". En Categories and De Interpretatione, Oxford, Clarendon Press.

Armstrong, D. M., 1978, Universals and Scientific Realism, 2 Vols. Cambridge, Cambridge University Press.

_—, 2009, "Reinhardt Grossmann's Grossmann Ontology". En

89. CSW: 403.

90. Cfr. Realism: 5, y E. Tegtmeier, Zeit und Existenz: Parmenideische Meditationen, Tübingen, Mohr Siebeck, 1997, p. 37.

91. Para más detalles, véase Cumpa (de próxima aparición).

Thémata. Revista de Filosofía N56 (2017) pp.: 213-234. 
Cumpa J. \& Tegtmeier, E. (2009).

Bergmann, G., 1964, Logic and Reality, Madison, University of Wisconsin Press.

- , 1967/2004, Realism: A Critique of Brentano and Meinong, Frankfurt, Ontos Verlag.

Cumpa, J. \& Tegtmeier, E., 2009, (PRvsSR), Phenomenological Realism Versus Scientific Realism: David M. Armstrong-Reinhardt Grossmann Metaphysical Correspondence, Ontos Verlag.

Ontos Verlag.

- 2010, Studies in the Ontology of Reinhardt Grossmann,

_ 2017, "Ordinary Objects and the Factualist Approach to Composition”. En Brewer, B. \& Cumpa, J. (eds), The Nature of Ordinary Objects, Cambridge University Press.

—- de próxima aparición, Eliminative Materialism and the Questions of Ontology: Reality, Categories, and Structure, Routledge.

Frege, G., 1960, "Function and Concept". En P. Geach and M. Black (ed.), Translations from the Philosophical Writings of Gottlob Frege, Blackwell.

Grossmann, R, 1963, (DL) "Sensory Intuition and the Dogma of Localization”. En Allaire, B. E. (ed.), Essays in Ontology, The Hage, Martinus Nijhoff.

University Press.

__, 1965, (SM) The Structure of Mind, Madison, Northwestern

—— 1969, (RF) Reflections on Frege's Philosophy, Evaston, Northwestern University Press, 1969.

University Press.

__ 1973, (OR) Ontological Reduction, Bloomington, Indiana

—— 1974a, "Bergmann's Ontology and the Principle of Acquaintance". En Gram M. S. and Klemke E. D. (eds.), The Ontological Turn: Studies in the Philosophy of Gustav Bergmann, Iowa City, University of Iowa Press.

— 1974b, Meinong, London, Routledge.

—_, 1975, "Perceptual Objects, Elementary Particles, and Emergent Properties". En Castañeda, H. N. (ed.), Action, Knowledge, and Reality: Critical Studies in Honour of Wilfrid Sellars, Bobbs-Merrill Company.

, 1977, "Introduction". En K. Twardowski, On the Content and the Object of Presentations, Martinus Nijhoff.

Bloomington, Indiana University Press.

London, Rouledge.

Thémata. Revista de Filosofía №56 (2017) pp.: 213-234. 
University Press.

1990, (FW) The Fourth Way: A Theory of Knowledge, Indiana

— 1992, (EW) The Existence of the World: An Introduction to Ontology, London, Routledge \& Kegan Paul.

_ , 2009, "A Legacy: Gustav Bergmann”. En Cumpa J. \& Tegtmeier, E. (eds.), Phenomenological Realism Versus Scientific Realism: David M. Armstrong-Reinhardt Grossmann Metaphysical Correspondence, Ontos Verlag.

__, 2009, "On the Simplicity of Universals". En Cumpa J. \& Tegtmeier, E. (2009).

—_, 2009, "Objections”. En Cumpa J. \& Tegtmeier, E. (2009).

_ 2009, "Comments on Armstrong's Universals: An Opinionated Introduction”. En Cumpa J. \& Tegtmeier, E. (2009). E. (2009).

__, 2009, "The Nature of Universals". En Cumpa J. \& Tegtmeier,

__ 2010, "Materialism and the New Folk Philosophy". En Cumpa, J. (2010).

Hempel, C. G. \& Oppenheim, P., 1948/1953, "Studies in the Logic of Explanation". En H. Feigl and M. Brodbeck (eds.), Readings in the Philosophy of Science, Appleton-Century-Crofts.

Hochberg, H., 1984, "Elementarism, Independence, and Ontology", en H. Hochberg, Logic, Ontology, and Language, München, Philosophia Verlag.

_ 2010, "Review of Phenomenological Realism Versus Scientific Realism”. En Dialectica, Vol. 64, No 3 .

Husserl, E., 1913/1962, Ideas Pertaining to a Pure Phenomenology and to a Phenomenological Philosophy, Collier Books.

__, 1970, Logical Investigations, 2 Vols., Humanity Press, Vol. I. Meinong, A., 1903/1913, "Gegenstandstheorie". In A. Meinong, Gesammelten Abhandlungen. Band. II, Leipzig.

—_, 1968-1978, Hume Studien I, en Haller, R. (ed.) Gesamtausgabe, 7 Vols., Verlaganstalt, Vol. I.

Russell, B., 1904, "Meinong's Theory of Complexes and Assumptions". In Mind, Vol. XIII.

__ 1912, The Problems of Philosophy, Home University Library.

— 1964, The Principles of Mathematics, Norton \& Company.

Sellars, W., 1963, Science, Perception and Reality, Routledge and Kegan Paul.

Stout, G. F., 1921-1922, "The Nature of Universals and Propositions". En Proceedings of the British Academy, Vol. X.

Tegtmeier, E., 1997, Zeit und Existenz: Parmenideische Meditationen, Tübingen, Mohr Siebeck. 
Wittgenstein, L., 1954, Philosophical Investigations, Oxford University Press.

— , 1961, Notebooks: 1914-1916, Blackwell.

Wolterstorff, N., 1971, "Qualities". En Landesman, C. (ed.), The Problem of Universals, Basic books. 